\title{
A diatom dataset and diatom-salinity inference model for southeast Australian estuaries and coastal lakes
}

\author{
Krystyna M. Saunders
}

Received: 3 April 2009/Accepted: 12 July 2010/Published online: 24 July 2010

(C) Springer Science+Business Media B.V. 2010

\begin{abstract}
To quantify the relationship between diatom species assemblages and the water chemistry of southeast Australian estuaries and coastal lakes, a new dataset of 81 modern diatom samples and water chemistry data was created. Three hundred and ninetynine species from 53 genera were identified in 36 samples from 32 coastal water bodies in eastern Tasmania and 45 samples from 13 coastal water bodies in southern Victoria. Multivariate statistical analyses revealed that the sampling sites were primarily distributed along salinity and nutrient gradients, and that salinity, nitrate + nitrite, phosphate and turbidity explained independent portions of variance in the diatom data. Species salinity optima and tolerances were determined and a diatom-salinity inference model (WAinv $r^{2}=0.72, r^{2}$ jack $=0.58$, RMSEP $=0.09 \log p p t$ ) was developed. This new information on diatom species' salinity preferences provides a useful tool for quantitatively reconstructing
\end{abstract}

Electronic supplementary material The online version of this article (doi:10.1007/s10933-010-9456-y) contains supplementary material, which is available to authorized users.

K. M. Saunders $(\bowtie)$

Institute of Geography and Oeschger Centre for Climate

Change Research, University of Bern, Bern 3012,

Switzerland

e-mail: krystyna.saunders@giub.unibe.ch

K. M. Saunders

Institute of Antarctic and Marine Studies, University

of TAS, Hobart, Tasmania 7001, Australia salinity changes over time from diatom microfossils preserved in the sediments of a range of estuaries and coastal lakes in southeast Australia. This is valuable for studies investigating long-term human impacts and climate change in the region.

Keywords Diatoms - Multivariate analyses · Transfer function - Australia - Water quality · Coast

\section{Introduction}

Diatoms are excellent indicators of environmental change in aquatic ecosystems as many taxa have distinct ecological requirements, limited tolerances to different water quality parameters and are highly sensitive to changes in their environment (Reid et al. 1995). Quantitative methods for relating diatom assemblages and water chemistry include modern calibration datasets and diatom inference models (i.e. transfer functions). These can be applied to sub-fossil diatom assemblages in sediment cores to reconstruct past water chemistry and to investigate past environmental and climate changes (Birks 1998). Much published work has been focused on inland lakes (e.g. Fritz et al. 1991; Bennion et al. 1996; Bigler and Hall 2003) but there are relatively few equivalent datasets for coastal lake and estuarine environments. However, some inference models have been developed and applied (e.g. Juggins 1992; Gehrels et al. 
2006; Ryves et al. 2004; Ellegaard et al. 2006; Horton et al. 2006; Weckström et al. 2004; Hassan et al. 2009). In Australia, diatom inference models for coastal lake and estuarine environments have so far been developed for relatively small geographic regions (e.g. Hodgson et al. 1996; Saunders et al. 2007, 2008; Taffs et al. 2008). Yet in some studies it is desirable to have diatom-based inference models that span a wider range of modern analogues, over a wider geographic area to better encompass the range of variability encountered in sub-fossil deposits. This is particularly important in regions that have experienced diverse and extensive environmental changes and/or human impacts, such as parts of coastal Australia. The development of larger, combined datasets has previously been undertaken in Africa (e.g. Gasse et al. 1995), Europe (e.g. Juggins 2004; Bennion 2010) and North America (e.g. Wilson et al. 1996).

Many Australian ecosystems have been highly modified since European settlement and with the majority of the population (85\%, SoE 2006) living in coastal regions, water quality is a key issue for environmental managers. Diatoms are recognised as one of the potentially most useful groups for water quality assessments in Australia (ANZECC 2000), but limited information regarding their ecological preferences has, to date, restricted their use. This paucity of data along Australia's extensive coastline needs to be addressed if diatoms are to be more widely applied to long term monitoring and management of the coastal zone.

This paper presents a new dataset quantifying the relationship between diatom species assemblages and the water chemistry of southeast Australian estuaries and coastal lakes. It extends a previously published dataset from Victoria (Saunders et al. 2008) with 36 sites from eastern Tasmania. The addition of the Tasmanian sites is useful because many of them have experienced only minor or transitory human impacts compared with their Victorian counterparts, and therefore provide additional potential analogues for diatom assemblages associated with pre-impact conditions in sediment cores. The specific aims of this study were to (a) provide species-level data on the composition and distribution of surface sediment diatom species assemblages in coastal water bodies in southeast Australia; (b) investigate and identify the environmental gradients influencing diatom species distributions and individual species ecological preferences; (c) develop inference models (i.e. transfer functions) based on the relationship between diatom species assemblages and environmental variables; and (d) discuss potential applications of the inference model to ecological and paleoecological investigations, with the aim of providing information to facilitate better environmental management.

\section{Materials and methods}

Study area

Rising sea levels last submerged the land bridge between Tasmania and Victoria approximately 13,000 years ago and the present coastline started forming near the end of the post-glacial marine transgression approximately 7-8,000 years ago. The coastline of the study area consists of quartzose beaches and dunes with a small number of calcareous beaches and dunes west of Wilson's Promontory. Geomorphological characteristics of the coastal estuarine habitats range from semi-enclosed bays that are characterize by marine waters with little fresh water inflow through to coastal water bodies that are brackish and rarely, if ever, connected to the ocean. The nature of these connections to the ocean determines the exchange of water and the salinity of these water bodies.

Climatologically southeast Australia has a variable, temperate climate (BOM 2009). Victoria's climate is typified by warm summers and mild winters and the climate is dominated by the El Niño Southern Oscillation (ENSO), with El Niño events often leading to drought and La Niña events sometimes resulting in flooding (BOM 2009). Tasmania's climate has four distinct seasons, with rainfall greatest in winter. The eastern half of the island is in the rain-shadow of the west and is more influenced by the ENSO. Victorian coastal systems experience seasonally variable rainfall, are infrequently flushed, not often stratified and tend to be more nitrogenlimited. Tasmanian coastal systems tend to receive more regular rainfall, are more commonly vertically stratified, frequently flushed and more likely to be phosphorus-limited (Davis and Koop 2006). The coastlines of both regions contain many coastal water bodies of high conservation value that range from small, closed lakes to large, open estuarine systems. 
These systems support the majority of the human population in both regions as well as key industries. They are consequently of significant environmental, societal and economic importance.

Sample collection

Thirty-six sites from 32 coastal water bodies in Tasmania were sampled and combined with the Victorian dataset (Saunders et al. 2008), which consisted of 45 sites from 13 coastal water bodies, creating a merged dataset of 81 sites. Sites spanned $>1,500 \mathrm{~km}$ of coastline $\left(37.50^{\circ} \mathrm{S}-42.92^{\circ} \mathrm{S}\right.$ and $144.4^{\circ} \mathrm{E}-149.7^{\circ} \mathrm{E}$, Fig. 1, Table 1$)$. Water chemistry was measured both in August 2004 and February 2005 in order to capture some of the seasonal variation in limnology. Salinity, temperature, $\mathrm{pH}$, turbidity and dissolved oxygen were measured in situ with a Horiba U-10 Water Quality Checker at $1 \mathrm{~m}$ water depth. Duplicate $10 \mathrm{~mL}$ water samples were collected for soluble reactive phosphate (referred to as phosphate hereafter), nitrate + nitrite and silicate analyses. Sample tubes were rinsed several times in lake/estuarine water at each site prior to collection. Samples were then frozen and analyzed using a Lachat Instrument (Injection Flow Analyzer) at the Commonwealth Scientific and Industrial Research Organization Marine and Atmospheric Laboratories, Hobart, within two months of collection. The autoanalyzer uses the principles of colorimetric analysis in a continuous flow system with all manipulations of the samples automated. Each analytical channel was calibrated using standards of known concentration (Eriksen 1997). The water chemistry measurements for each site were combined to determine a mean value for each variable for the statistical analyses.

Surface sediment samples (top $1 \mathrm{~cm}$ ), which provide a spatially and temporally integrated sample of the diatom communities at each site (Lim et al. 2007), were collected from approximately $1 \mathrm{~m}$ water depth using a hand-operated gravity corer (Glew 1991). Surface sediment samples were collected at the end of the water sampling period (February 2005) to ensure that the diatom species identified more closely corresponded with the water chemistry measured. The number of samples taken from each water body was variable (ranging from 1 to 14 sites), depending on size and complexity, to try and account for spatial variability in diatom habitats and water chemistry.
Diatom preparation and analysis

Diatom samples were prepared following standard methods (Battarbee 1986). At least 400 frustules per sample were counted using oil immersion at $1000 \times$ magnification on a Zeiss 20 light microscope. The relative abundance of all species (including unidentified forms) was recorded as a percentage of the total number of frustules counted (Battarbee et al. 2001). Taxonomy was principally based on Australian taxonomic literature (i.e. Hodgson et al. 1997; John 1983; Sonneman et al. 2000) and datasets (i.e. Hodgson et al. 1996; Saunders et al. 2007; Taffs 2005), with additional reference to European coastal diatom taxonomic references (e.g. Witkowski et al. 2000). As diatom taxonomy requires further development in this region (see Vyverman et al. 1995) all taxa were photographed and are archived with the author.

\section{Statistical analyses}

Multivariate statistical analyses were used to identify major environmental gradients, explore diatom-environment relationships and identify environmental variables that explained independent portions of the variance in the diatom data.

Prior to statistical analyses, each environmental variable was checked for skewness, and nutrients and turbidity were $\log (x+1)$ transformed. Principal Components Analysis (PCA) was performed on the environmental data to determine the major environmental gradients. Detrended Correspondence Analysis (DCA) with detrending by segments and downweighting of rare species was performed on the species data to establish whether species distribution was unimodal or linear. As gradient lengths were greater than 2 standard deviation units, unimodal ordination techniques were used (ter Braak 1995). Species data were $\log (x+1)$ transformed in an attempt to stabilize the variance in the dataset (Birks et al. 2001).

A series of Canonical Correspondence Analyses (CCA) were performed with scaling focused on interspecies distances, biplot scaling and downweighting of rare species. Variance Inflation Factors (VIFs) were identified and any environmental variables with VIFs $>20$ were removed. A series of CCAs of each environmental variable alone was performed, followed by CCAs of individual environmental variables with the remainder as covariables (i.e. forward 
Fig. 1 a Location of study area; $\mathbf{b}$ location of sampling sites in Victoria and c Tasmania with specific sites numbered. See Supplementary Material for specific locations and details of sampling sites

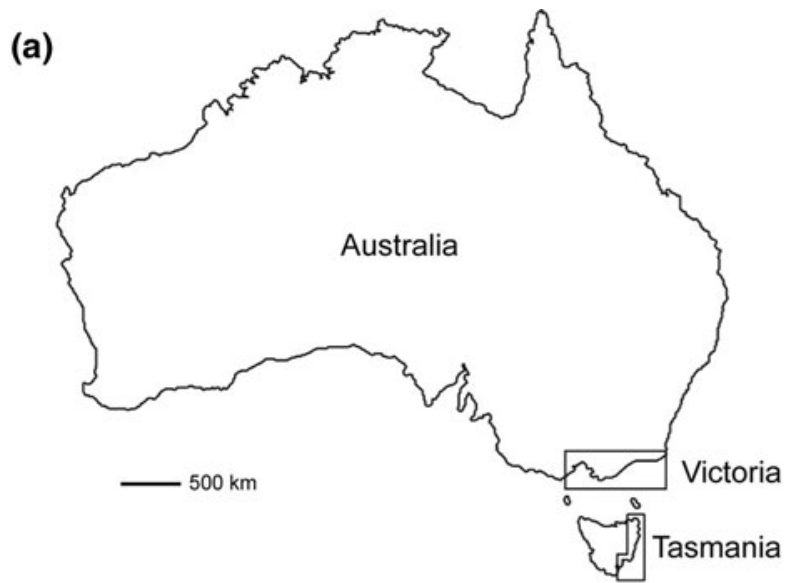

(b)

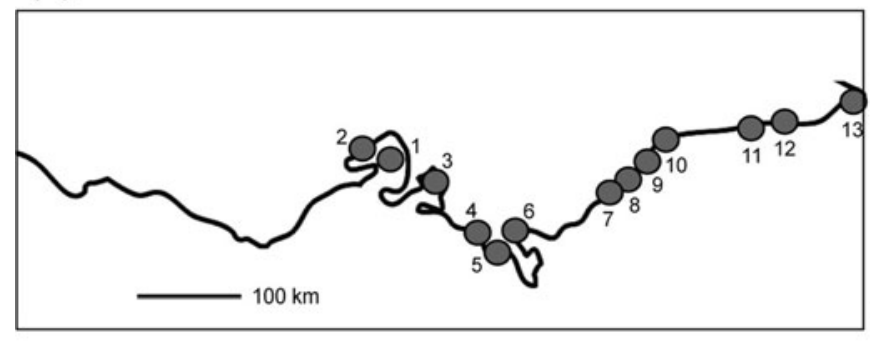

(c)

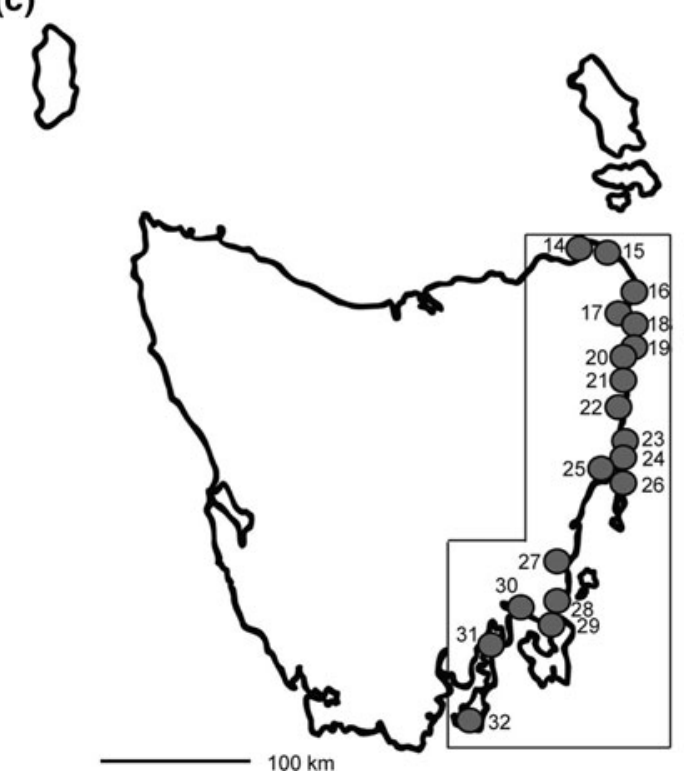

$1 \mathrm{P} 3, \mathrm{P} 5, \mathrm{P} 6, \mathrm{P} 7$,

$\mathrm{P} 9, \mathrm{P} 10, \mathrm{P} 12, \mathrm{P} 13$.

P14, P15, P16, P17

2 P4

3 WPB1, WPB2, WPB3, WPB4

$4 \mathrm{Al} 1, \mathrm{Al} 2, \mathrm{Al} 3$

$5 \mathrm{SI} 2$

$6 \mathrm{Cl} 1, \mathrm{Cl} 2, \mathrm{Cl} 3$

7 GL10, GL11

8 GL8, GL12,

9 GL1, GL2, GL3, GL4

GL5, GL6

10 TAM1

11 SYD1

12 LT1, LT2, LT3, LT4

13 MC1, MC2, MC3,

MC4, MC5

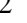




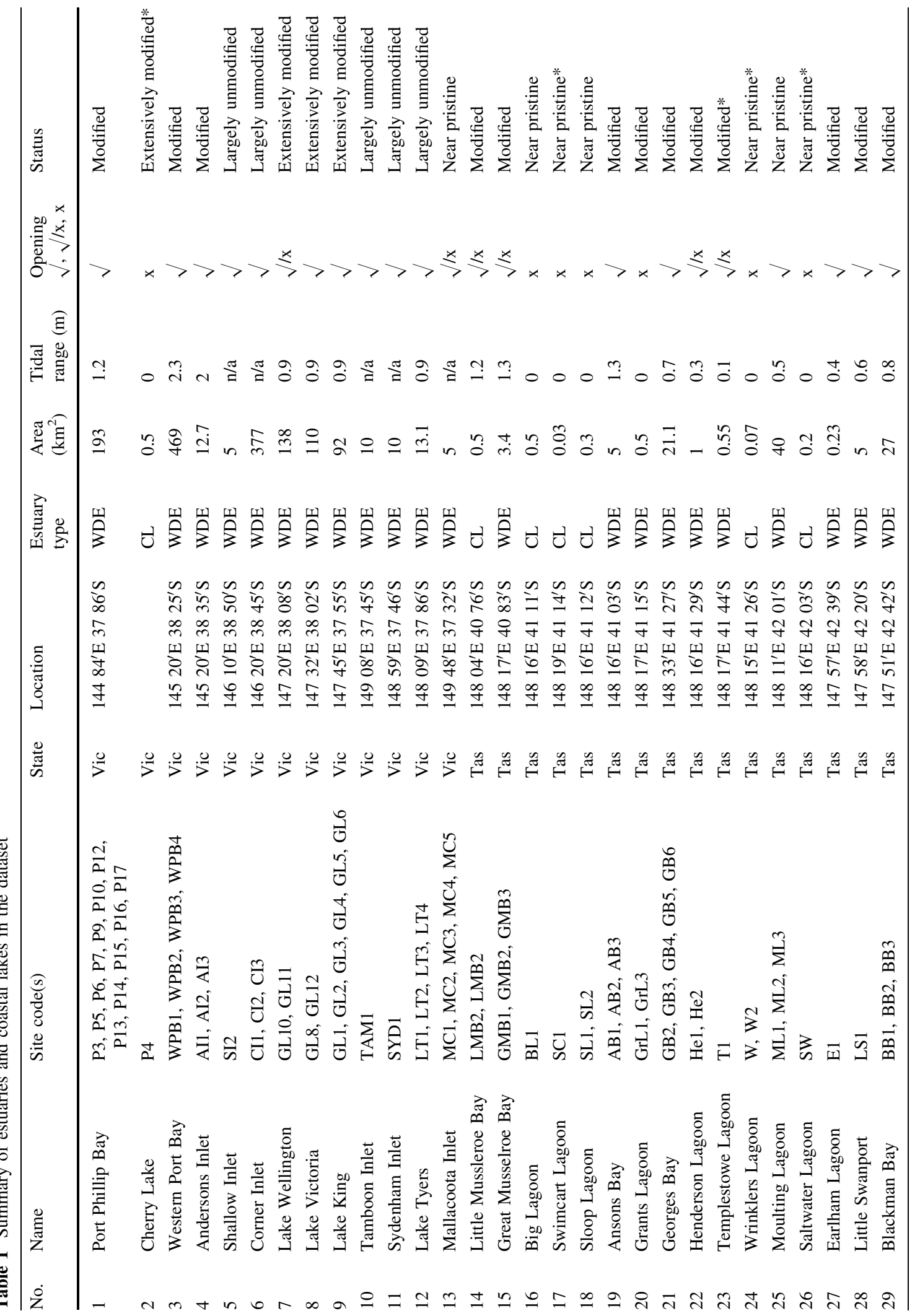




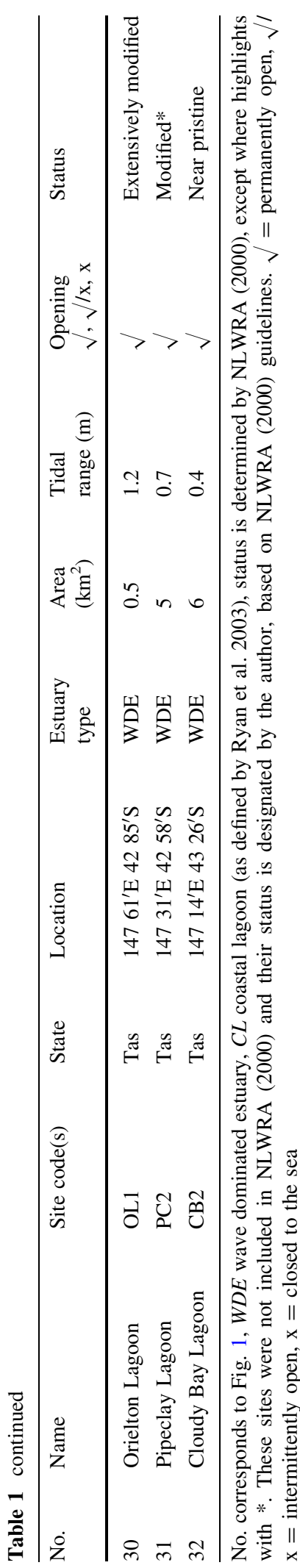

et al. 1992), was used to determine which environmental variable was most suitable for transfer function development (Birks 1998). All ordinations were performed using the software program $\mathrm{R}$ (R Development Core Team 2006) and CANOCO 4.5 for Windows (ter Braak and Smilauer 2002).

Species ecological preferences (i.e. optima and tolerances) were determined using simple Weighted Averaging (WA) on untransformed species data. Transfer functions were developed using simple WA with inverse and classical deshrinking and with/without tolerance downweighting, and Weighted Averaging Partial Least Squares (WAPLS) to determine which technique led to the best performing transfer function. Performance was assessed using leave-one-out cross validation (i.e. jackknifing, Birks 1998). When assessing which model to use, a WAPLS model with a higher number of components was only accepted if there was $\geq 5 \%$ improvement in RMSEP over alternatives with less components (Birks 1998). Sites were selected for deletion based on visual inspection of the residual difference between predicted and observed salinity, with outliers removed until model performance did not improve. Species ecological preferences and transfer functions were determined using the software program C2 version 1.4 (Juggins 2003).

\section{Results}

Environmental data and diatom assemblages

The water chemistry data for Tasmanian and Victorian estuaries and coastal lakes that were sampled are summarized and outlined in Tables 2 and 3. Salinity ranged from near fresh to hypersaline, while nutrient concentrations ranged from oligotrophic to eutrophic. In general, the Victorian sites had poorer water quality, as represented by higher nutrient and lower dissolved oxygen concentrations than the Tasmanian sites. Information for each site is provided in the Supplementary material.

PCA of the environmental data showed that the first two axes explained $42 \%$ of the variation in the environmental data. Variation along the first axis was explained by nitrate + nitrite, while variation along the second axis was explained by $\mathrm{pH}$ (Fig. 2).

In total, 399 diatom species were identified from 53 genera. Of these, 186 species $(47 \%)$ were common 
Table 2 Summary of water quality data for Tasmanian and Victorian sites

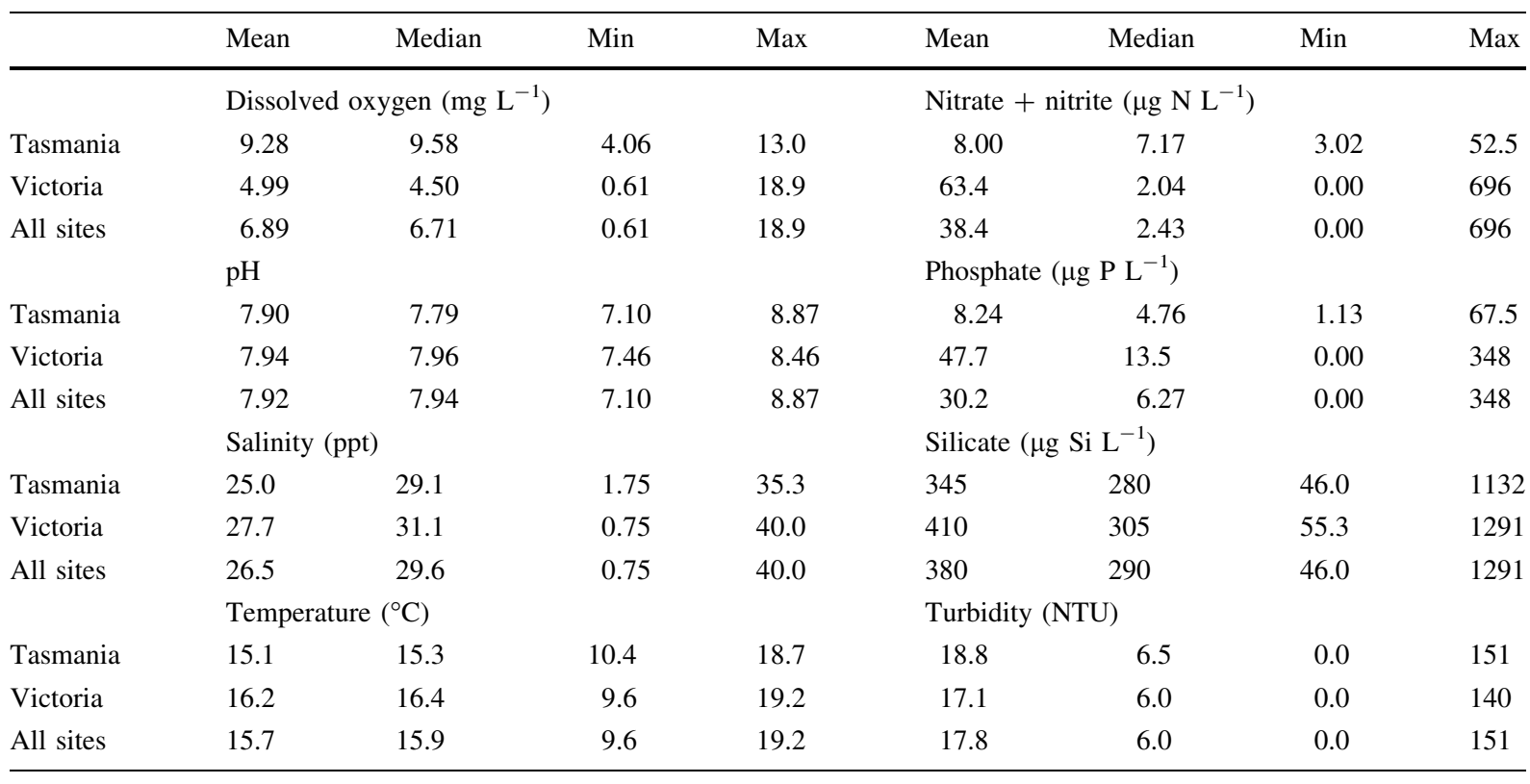

$N$ nitrate-nitrite, $P$ phosphate, $S i$ silicate

between Tasmania and Victoria. Most of the diatom taxa were benthic. Thirty-five percent were rare (occurring only once), while $46 \%$ occurred at low $(<1 \%)$ relative abundance. Thirty-nine species were abundant (i.e. $\geq 10 \%$ maximum relative abundance in one or more samples, Table 4), while 29 were widespread (i.e. occurring in $\geq 20$ sites). Opephora guenter-grassii (Witkowski and Lange-Bertalot) Sabbe and Vyverman, and Planothidium hauckianium agg. (Kützing) Round and Bukhtiyarova were the most abundant and widespread: Opephora guentergrassii occurred in $67(83 \%)$ sites with $47.6 \%$ maximum relative abundance; Planothidium hauckianium agg. occurred in $78(96 \%)$ sites with $57.5 \%$ maximum relative abundance. For the statistical analyses, only those species occurring with a maximum relative abundance $\geq 2 \%$ and in $\geq 2$ samples were included, resulting in a total of 141 taxa. All taxa are listed in the Supplementary material (including their mean and maximum relative abundances).

Multivariate analyses: diatom-environment relationships

An initial CCA indicated the environmental data explain $16.3 \%$ of the variation in the diatom data (Table 5). As all VIFs were $<10$, all environmental variables were retained. Salinity was correlated to axis 1 , while the remaining variables were correlated to both axis 1 and axis 2 (Fig. 3). Nitrate + nitrite, phosphate, salinity and turbidity explained independent portions of the variance in the diatom data (as determined by forward selection) and CCA of these variables indicated they explained $10 \%$ of the variation in the diatom data (Fig. 3, Table 5). Variance partitioning indicated that $7.3 \%$ of the variation in the diatom data was due to these environmental variables alone and the total interaction between them was $1.3 \%$ (Fig. 4). Salinity explained the most variation $(2.6 \%)$, followed by phosphate $(2.0 \%)$, nitrate + nitrite $(1.7 \%)$ and turbidity $(1.0 \%)$. The greatest interaction occurred between nitrate + nitrite and the other variables $(0.6 \%)$.

To determine which, if any, of the environmental variables were suitable for transfer function development, DCCA was performed with axis 1 constrained to each environmental variable in turn, with axis 2 left unconstrained. DCCA indicated that salinity was suitable (i.e. $\lambda_{1} / \lambda_{2}=0.6$ ).

Species optima and tolerances and model development

Simple WA was used to determine species optima and tolerances for salinity. Information for all species 
Table 3 Water chemistry results for sites in the dataset

\begin{tabular}{|c|c|c|c|c|c|c|c|c|c|c|}
\hline Code & $\begin{array}{l}\text { Latitude } \\
\left({ }^{\circ} \mathrm{S}\right)\end{array}$ & $\begin{array}{l}\text { Longitude } \\
\left({ }^{\circ} \mathrm{E}\right)\end{array}$ & 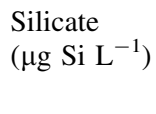 & $\begin{array}{l}\text { Phosphate } \\
\left(\mu g \mathrm{P} \mathrm{L}^{-1}\right)\end{array}$ & $\begin{array}{l}\text { Nitrate }+ \text { nitrite } \\
\left(\mu \mathrm{g} \mathrm{N} \mathrm{L}^{-1}\right)\end{array}$ & $\begin{array}{l}\text { Salinity } \\
\text { (ppt) }\end{array}$ & $\mathrm{pH}$ & $\begin{array}{l}\text { Temp. } \\
\left({ }^{\circ} \mathrm{C}\right)\end{array}$ & $\begin{array}{l}\text { Turbidity } \\
\text { (NTU) }\end{array}$ & $\begin{array}{l}\text { Dissolved } \\
\text { oxygen } \\
\left(\mathrm{mg} \mathrm{L}^{-1}\right)\end{array}$ \\
\hline $\mathrm{AB} 1$ & -41.0625 & 148.289 & 306.51 & 3.79 & 8 & 31.9 & 8.08 & 16.2 & 2 & 10.1 \\
\hline $\mathrm{AB} 2$ & -41.0536 & 148.27 & 365.88 & 67.46 & 2.28 & 30.3 & 7.77 & 16.1 & 151 & 5.6 \\
\hline AB3 & -41.0366 & 148.285 & 298.04 & 4.8 & 1.27 & 30 & 8.13 & 15.7 & 1 & 10.45 \\
\hline AI1 & -38.6734 & 145.797 & 1290.96 & 81.72 & 389.25 & 11.4 & 7.89 & 17.6 & 73 & 4.52 \\
\hline $\mathrm{AI} 2$ & -38.6614 & 145.783 & 864.33 & 42.78 & 164.78 & 23.1 & 7.84 & 16.3 & 16 & 0.79 \\
\hline $\mathrm{AI} 3$ & -38.6617 & 145.77 & 422.3 & 19.17 & 33.12 & 30 & 7.88 & 16.8 & 23 & 5.42 \\
\hline BB1 & -42.8466 & 147.844 & 48.37 & 2.59 & 2.43 & 33.6 & 8.11 & 15.5 & 0 & 9.19 \\
\hline BB2 & -42.8905 & 147.809 & 58.28 & 1.13 & 2.19 & 33.3 & 8.06 & 15.6 & 1 & 9.4 \\
\hline BB3 & -42.8905 & 147.809 & 93.29 & 12.82 & 52.5 & 33.7 & 8.23 & 17.8 & 1 & 11.37 \\
\hline BL1 & -41.1847 & 148.267 & 639.01 & 2.96 & 3.33 & 11.6 & 7.1 & 11 & 8 & 10.25 \\
\hline CB2 & -42.959 & 147.524 & 92.98 & 2.03 & 4.08 & 32.7 & 7.91 & 13 & 8 & 5.4 \\
\hline CI1 & -38.7008 & 146.455 & 104.55 & 1.66 & 2.12 & 34.3 & 8.03 & 17.2 & 3.5 & 0.61 \\
\hline CI2 & -38.691 & 146.336 & 211.59 & 13.71 & 0.62 & 33.8 & 8.13 & 17.4 & 32.5 & 1.3 \\
\hline CI3 & -38.8136 & 146.267 & 55.26 & 1.75 & 0.57 & 34.6 & 8.46 & 19 & 1.5 & 1.58 \\
\hline E1 & -42.6545 & 147.939 & 83.6 & 2.59 & 2.19 & 33.6 & 7.98 & 14.4 & 7 & 7.9 \\
\hline GB2 & -41.2952 & 148.273 & 204.95 & 3.69 & 6.58 & 31.4 & 7.81 & 12.5 & 8 & 7.69 \\
\hline GB3 & -41.3112 & 148.27 & 161.69 & 2.65 & 2.75 & 32.1 & 7.91 & 13.9 & 0 & 9.65 \\
\hline GB4 & -41.3316 & 148.248 & 258.6 & 7.03 & 4.9 & 33 & 7.73 & 14 & 7 & 9.9 \\
\hline GB5 & -41.3254 & 148.298 & 175.09 & 6.01 & 5.17 & 31.8 & 7.85 & 13.7 & 7 & 9.3 \\
\hline GB6 & -41.3035 & 148.315 & 151.59 & 10.13 & 15.13 & 32.6 & 7.46 & 13.9 & 5 & 8.82 \\
\hline GL1 & -37.8788 & 148.004 & 270.28 & 29.92 & 0.08 & 24.7 & 8.13 & 16 & 1.5 & 3.44 \\
\hline GL2 & -37.8837 & 147.978 & 437.71 & 2.25 & 0.4 & 24.6 & 8.09 & 15.4 & 2.5 & 3.18 \\
\hline GL3 & -37.8826 & 147.975 & 444.49 & 2.15 & 0.04 & 23.9 & 8.06 & 15.7 & 6 & 3.33 \\
\hline GL4 & -37.8839 & 147.89 & 282.24 & 2.66 & 0.01 & 21.7 & 8.09 & 15.4 & 0.5 & 3.32 \\
\hline GL5 & -37.8986 & 147.855 & 289.8 & 6.29 & 0.02 & 21.3 & 8.16 & 15.5 & 1.5 & 3.32 \\
\hline GL6 & -37.9276 & 147.711 & 550.73 & 13.98 & 1.31 & 20.9 & 8.07 & 14.7 & 0.5 & 1.53 \\
\hline GL8 & -37.8939 & 147.684 & 337.54 & 13.5 & 0.29 & 21.4 & 8.16 & 16.3 & 1 & 3.3 \\
\hline GL10 & -37.8876 & 147.677 & 1184.42 & 26.1 & 13.33 & 12 & 8.04 & 16 & 5 & 3.02 \\
\hline GL11 & -37.8823 & 147.718 & 1080.08 & 10.16 & 14.52 & 9.6 & 8 & 15.8 & 9 & 3.27 \\
\hline GL12 & -37.8823 & 147.718 & 474.44 & 25.52 & 0.1 & 20.9 & 8.14 & 16.7 & 4 & 6.2 \\
\hline GrL1 & -41.2539 & 148.287 & 320.34 & 1.55 & 0 & 29.1 & 7.88 & 14.7 & 5 & 10 \\
\hline GrL3 & -41.2535 & 148.298 & 445.39 & 2.17 & 0 & 27.8 & 7.67 & 14.9 & 29 & 8.72 \\
\hline GMB1 & -40.8324 & 148.176 & 731.91 & 8.52 & 33.46 & 14.5 & 7.94 & 16.7 & 11 & 10.7 \\
\hline GMB2 & -40.8361 & 148.176 & 236.71 & 5.65 & 16.49 & 20.3 & 8.22 & 18.4 & 5 & 12.95 \\
\hline GMB3 & -40.8399 & 148.174 & 390.08 & 5.23 & 16.07 & 18.4 & 8.11 & 18 & 6 & 11.62 \\
\hline $\mathrm{He} 1$ & -41.4789 & 148.252 & 194 & 6.3 & 9.49 & 29.6 & 7.89 & 14.4 & 26 & 9.2 \\
\hline $\mathrm{He} 2$ & -41.5051 & 148.269 & 1132.13 & 15.43 & 6.04 & 23.6 & 7.85 & 13.7 & 10 & 10.25 \\
\hline LMB1 & -40.7657 & 148.035 & 286.42 & 2.2 & 3.96 & 29.1 & 8.26 & 18.3 & 1 & 6.05 \\
\hline LMB2 & -40.7659 & 148.034 & 292.38 & 5 & 3.17 & 27.2 & 8.12 & 18.7 & 0 & 8.72 \\
\hline LS1 & -41.9949 & 147.989 & 118.51 & 4.12 & 2.87 & 32.6 & 7.94 & 14.2 & 3 & 5.95 \\
\hline LT1 & -37.8532 & 148.084 & 185.02 & 11.18 & 1.86 & 35.6 & 7.75 & 16.4 & 5.5 & 2.45 \\
\hline LT2 & -37.8529 & 148.063 & 149.67 & 4.63 & 1.5 & 36.1 & 7.65 & 16.5 & 18 & 4.77 \\
\hline LT3 & -37.8357 & 148.074 & 322.63 & 2.06 & 2.4 & 35.8 & 7.69 & 16.3 & 1.5 & 4.03 \\
\hline
\end{tabular}


Table 3 continued

\begin{tabular}{|c|c|c|c|c|c|c|c|c|c|c|}
\hline Code & $\begin{array}{l}\text { Latitude } \\
\left({ }^{\circ} \mathrm{S}\right)\end{array}$ & $\begin{array}{l}\text { Longitude } \\
\left({ }^{\circ} \mathrm{E}\right)\end{array}$ & $\begin{array}{l}\text { Silicate } \\
\left(\mu \mathrm{g} \mathrm{Si} \mathrm{L} \mathrm{L}^{-1}\right)\end{array}$ & $\begin{array}{l}\text { Phosphate } \\
\left(\mu g \mathrm{P} \mathrm{L}^{-1}\right)\end{array}$ & $\begin{array}{l}\text { Nitrate }+ \text { nitrite } \\
\left(\mu \mathrm{g} \mathrm{N} \mathrm{L}^{-1}\right)\end{array}$ & $\begin{array}{l}\text { Salinity } \\
\text { (ppt) }\end{array}$ & $\mathrm{pH}$ & $\begin{array}{l}\text { Temp. } \\
\left({ }^{\circ} \mathrm{C}\right)\end{array}$ & $\begin{array}{l}\text { Turbidity } \\
\text { (NTU) }\end{array}$ & $\begin{array}{l}\text { Dissolved } \\
\text { oxygen } \\
\left(\mathrm{mg} \mathrm{L}^{-1}\right)\end{array}$ \\
\hline LT4 & -37.8123 & 148.057 & 465.66 & 11.97 & 0.36 & 27.7 & 7.64 & 17.5 & 6 & 4.5 \\
\hline MC1 & -37.5099 & 149.692 & 842 & 2.69 & 0.16 & 25.6 & 7.81 & 16.5 & 8 & 5.53 \\
\hline MC2 & -37.4993 & 149.702 & 569.63 & 5.22 & 2.58 & 25.3 & 7.84 & 16.9 & 7 & 6.91 \\
\hline MC3 & -37.5327 & 149.74 & 242.19 & 3.31 & 0.85 & 24.7 & 7.93 & 15.7 & 3 & 6 \\
\hline MC4 & -37.5367 & 149.742 & 137.17 & 4.14 & 2.04 & 25.8 & 7.98 & 15.7 & 6 & 6.71 \\
\hline MC5 & -37.511 & 149.702 & 469.13 & 1.35 & 0.02 & 26.2 & 7.91 & 16.8 & 1.5 & 5.33 \\
\hline ML1 & -41.2678 & 148.192 & 274.42 & 11.63 & 1.23 & 29 & 8.22 & 15.7 & 2 & 11.46 \\
\hline ML2 & -42.0285 & 148.221 & 685.36 & 11.55 & 1.16 & 11.2 & 7.98 & 15.9 & 36 & 10.15 \\
\hline ML3 & -41.9924 & 148.245 & 545.84 & 28.79 & 0.19 & 6.5 & 7.76 & 15.6 & 109 & 7.61 \\
\hline OL1 & -42.959 & 147.524 & 98.29 & 7.12 & 1.06 & 35.3 & 8.19 & 18.2 & 150 & 4.06 \\
\hline P3 & -37.86 & 144.866 & 183.38 & 68.33 & 28.25 & 33 & 7.63 & 9.6 & 6 & 2.99 \\
\hline P4 & -37.8639 & 144.838 & 973.33 & 100.57 & 434.17 & 0.8 & 8.39 & 16.3 & 139.665 & 2.22 \\
\hline P5 & -37.8746 & 144.817 & 84.72 & 264.43 & 1.55 & 34.1 & 8 & 16.9 & 12.5 & 1.64 \\
\hline P6 & -37.9543 & 144.721 & 304.65 & 199.27 & 696.14 & 33.7 & 7.97 & 17.1 & 47 & 1.41 \\
\hline P7 & -37.9718 & 144.699 & 264.62 & 195.11 & 586.14 & 34.2 & 8.01 & 17.3 & 36.5 & 1.42 \\
\hline P9 & -38.0292 & 144.563 & 154.45 & 347.61 & 25.04 & 34.7 & 8.05 & 19 & 10 & 1.6 \\
\hline P10 & -38.0865 & 144.424 & 148.63 & 140.13 & 0.95 & 36.5 & 8.22 & 19.2 & 7.25 & 1.85 \\
\hline P12 & -38.1599 & 144.455 & 261.99 & 64.33 & 4.76 & 35.6 & 8.11 & 17 & 2 & 7.71 \\
\hline P13 & -38.1164 & 144.671 & 62.48 & 48.85 & 1.93 & 34.9 & 7.96 & 15 & 0 & 6.03 \\
\hline P14 & -38.1616 & 144.714 & 225.53 & 161.75 & 4.31 & 40 & 8.05 & 11.8 & 131 & 11.93 \\
\hline P15 & -38.369 & 144.858 & 258.69 & 55.97 & 1.94 & 33.7 & 7.92 & 17 & 1 & 7.77 \\
\hline P16 & -38.0992 & 144.782 & 331.19 & 59.1 & 97.35 & 33.5 & 7.67 & 13.8 & 2 & 18.9 \\
\hline P17 & -38.0864 & 145.463 & 418.99 & 55.6 & 189.33 & 31.1 & 7.94 & 17 & 5 & 9.58 \\
\hline PC2 & -42.959 & 147.524 & 45.97 & 3.62 & 1.17 & 33.2 & 8.02 & 17.3 & 3 & 8.49 \\
\hline $\mathrm{SC} 1$ & -41.2299 & 148.282 & 452.35 & 15.16 & 2.03 & 1.8 & 7.27 & 10.4 & 19 & 11.15 \\
\hline SI2 & -38.8423 & 146.151 & 86.14 & 16.91 & 4.71 & 33.9 & 7.92 & 15.6 & 24.5 & 5.57 \\
\hline SL1 & -41.2091 & 148.272 & 110.78 & 4.03 & 12.82 & 19.4 & 7.79 & 11.2 & 0 & 10.2 \\
\hline SL2 & -41.2043 & 148.26 & 304.7 & 3.75 & 11.29 & 4.9 & 7.29 & 12.5 & 14 & 10.21 \\
\hline SW & -41.4401 & 148.27 & 940.9 & 13.07 & 0.14 & 20.2 & 8.87 & 15.3 & 5 & 12.65 \\
\hline SYD1 & -37.7625 & 148.971 & 355.3 & 3.79 & 6.35 & 16.4 & 7.63 & 15.6 & 14 & 9.77 \\
\hline TAM1 & -37.7434 & 149.136 & 891.7 & 5.87 & 1.08 & 19 & 7.46 & 15.1 & 10 & 8.46 \\
\hline $\mathrm{T} 1$ & -41.7238 & 148.272 & 1068.1 & 4.71 & 1.72 & 28.6 & 7.75 & 15.3 & 23 & 9.5 \\
\hline W1 & -41.4401 & 148.27 & 152.67 & 3.21 & 19.94 & 13.8 & 7.59 & 13.7 & 8 & 10.4 \\
\hline W2 & -41.4401 & 148.27 & 670.69 & 4.31 & 1.17 & 14 & 7.62 & 18.1 & 4 & 8.96 \\
\hline WPB1 & -38.4094 & 145.419 & 274.37 & 1.55 & 7.19 & 33.3 & 7.9 & 17.5 & 7.5 & 5.31 \\
\hline WPB2 & -38.3082 & 145.52 & 835.57 & 11.83 & 71.01 & 32 & 7.76 & 18 & 44.5 & 4.52 \\
\hline WPB3 & -38.217 & 145.376 & 461.25 & 4.74 & 57.04 & 32.1 & 7.79 & 18.9 & 22.5 & 6.44 \\
\hline WPB4 & -38.3755 & 145.221 & 84.24 & 0 & 0 & 34 & 7.68 & 12.4 & 9 & 14.98 \\
\hline
\end{tabular}

is provided in the Supplementary material and is summarized in Fig. 5. There were clear differences in the relative abundance of the most common diatoms along the salinity gradient (Fig. 6). For example,
Diatomella cf. balfouriana Greville was most abundant at low ( $<5 \mathrm{ppt})$ salinity sites, Rhopalodia acuminata Krammer and Cyclotella choctawhatcheeana Prasad were most abundant at brackish (5-30 ppt) 
Table 4 Number of occurrences $(\mathrm{N})$, effective number of occurrences (Hill's N ${ }^{2}$ ) (Hill 1973), maximum relative abundance, and weighted averaging salinity optima (WA opt) and tolerances (WA tol) of the most dominant (i.e. maximum relative abundance $\geq 10 \%$ ) diatom taxa

\begin{tabular}{|c|c|c|c|c|c|c|}
\hline Taxa & Code & $\mathrm{N}$ & $\mathrm{N} 2$ & $\operatorname{Max}$ & $\begin{array}{l}\text { WA Opt } \\
(\mathrm{ppt})\end{array}$ & $\begin{array}{l}\text { WA Tol } \\
\text { (ppt) }\end{array}$ \\
\hline Achnanthes angustata & OPE3 & 53 & 37.36 & 17.12 & 27.95 & 7.30 \\
\hline Achnanthes angustata var. 1 & OPE6 & 57 & 40.49 & 21.14 & 28.71 & 6.10 \\
\hline Achnanthes brevipes var. intermedia & EPI1 & 8 & 3.41 & 10.87 & 23.69 & 5.23 \\
\hline Achnanthes sp. 1 & PLAhau3 & 18 & 9.57 & 35.71 & 25.68 & 11.41 \\
\hline Actinocyclus subtilis & CEN10 & 4 & 2.73 & 40.33 & 31.03 & 3.48 \\
\hline Amphora acutiuscula & AMPcof & 70 & 51.68 & 12.17 & 27.55 & 7.24 \\
\hline Amphora exigua & AMPcof2 & 44 & 29.48 & 10.87 & 29.41 & 5.97 \\
\hline Amphora sp. 1 & AMP3 & 21 & 13.95 & 15.21 & 28.23 & 7.83 \\
\hline Catenula adherens & AMP1 & 68 & 52.93 & 39.56 & 28.21 & 6.67 \\
\hline Cocconeis peltoides & $\mathrm{ACH} 3$ & 53 & 40.93 & 15.88 & 27.15 & 7.08 \\
\hline Cocconeis placentula & COCpla & 51 & 33.68 & 51.00 & 26.95 & 7.83 \\
\hline Cocconeis scutellum & COCscu & 57 & 39.27 & 30.47 & 27.07 & 8.27 \\
\hline Cocconeis scutellum var. 1 & COCdis & 54 & 34.86 & 56.22 & 29.93 & 6.57 \\
\hline Cocconeis sp. 1 & UNK107 & 21 & 15.24 & 16.50 & 31.82 & 3.13 \\
\hline Coscinodiscus centralis & CEN1 & 43 & 21.97 & 54.25 & 29.88 & 6.59 \\
\hline Cyclotella choctawhatcheeana & CYCstr2 & 33 & 19.31 & 29.01 & 27.10 & 7.49 \\
\hline Cyclotella striata & CYCstr & 25 & 15.88 & 12.80 & 25.97 & 6.96 \\
\hline Diatomella cf. balfouriana & UNK37b & 3 & 2.45 & 51.38 & 10.94 & 12.00 \\
\hline Diploneis cf. domblitlensis & FAL2 & 20 & 10.84 & 14.88 & 30.58 & 5.76 \\
\hline Fragilaria ellipta & CEN5 & 32 & 19.70 & 14.50 & 28.51 & 6.70 \\
\hline Grammatophora angulosa var. angulosa & GRA3 & 6 & 2.78 & 16.75 & 31.71 & 5.25 \\
\hline Grammatophora marina & GRAoce & 39 & 24.19 & 41.87 & 27.94 & 6.36 \\
\hline Gyrosigma balticum & GYRbal & 40 & 24.84 & 12.56 & 30.47 & 5.54 \\
\hline Mastogloia pusilla & $\operatorname{MAS} 2 \mathrm{a}$ & 14 & 6.54 & 24.88 & 31.87 & 3.04 \\
\hline Melosira nummuloides & MEL1 & 13 & 7.81 & 16.46 & 30.10 & 8.07 \\
\hline Navicula cf. lusoria & $\mathrm{ACH} 14$ & 15 & 11.36 & 21.16 & 33.42 & 2.10 \\
\hline Navicula perminuta & NAVper & 59 & 43.75 & 14.29 & 28.20 & 6.62 \\
\hline Navicula recens & NAV1 & 62 & 45.75 & 14.07 & 28.36 & 6.97 \\
\hline Navicula salinarum var. salinarum & NAV3 & 49 & 34.03 & 11.47 & 29.18 & 6.57 \\
\hline Nitzschia cf. valdestriata & NITval & 54 & 35.81 & 14.85 & 29.36 & 5.94 \\
\hline Opephora guenter-grassii & OPEgue & 67 & 47.91 & 47.65 & 28.08 & 6.49 \\
\hline Opephora pacifica & OPEbur2 & 3 & 2.28 & 20.29 & 31.91 & 3.97 \\
\hline Opephora pacifica var. 1 & OPEbur & 58 & 42.36 & 64.22 & 27.68 & 6.65 \\
\hline Paralia sp. 1 & PARsul & 33 & 24.77 & 12.37 & 28.66 & 5.89 \\
\hline Planothidium delicatulum & PLAdel & 64 & 50.34 & 28.75 & 27.16 & 6.75 \\
\hline Planothidium delicatulum var. 1 & PLAdel3 & 26 & 19.72 & 12.90 & 29.20 & 5.65 \\
\hline Planothidium hauckianium & PLAhau & 78 & 60.71 & 57.53 & 28.58 & 6.40 \\
\hline Rhopalodia acuminata & RHA3 & 42 & 25.96 & 34.15 & 26.40 & 6.45 \\
\hline Trachyspenia australis var. australis & VIK8a & 10 & 6.15 & 12.20 & 31.82 & 3.73 \\
\hline
\end{tabular}

sites, while Cocconeis scutellum Ehrenberg var. 1 and Navicula cf. lusoria Giffen were more abundant at high ( $\geq 33$ ppt) salinity sites.

Simple WA with inverse deshrinking resulted in the best performing salinity transfer function. Six sites were identified as outliers and removed. The final model consisted of 75 sites $\left(r^{2}=0.72\right.$, $r^{2}$ jack $=0.58, \quad$ RMSEP $=0.09 \log$ ppt, Table 6, Fig. 7). WAPLS did not significantly improve transfer function performance. 

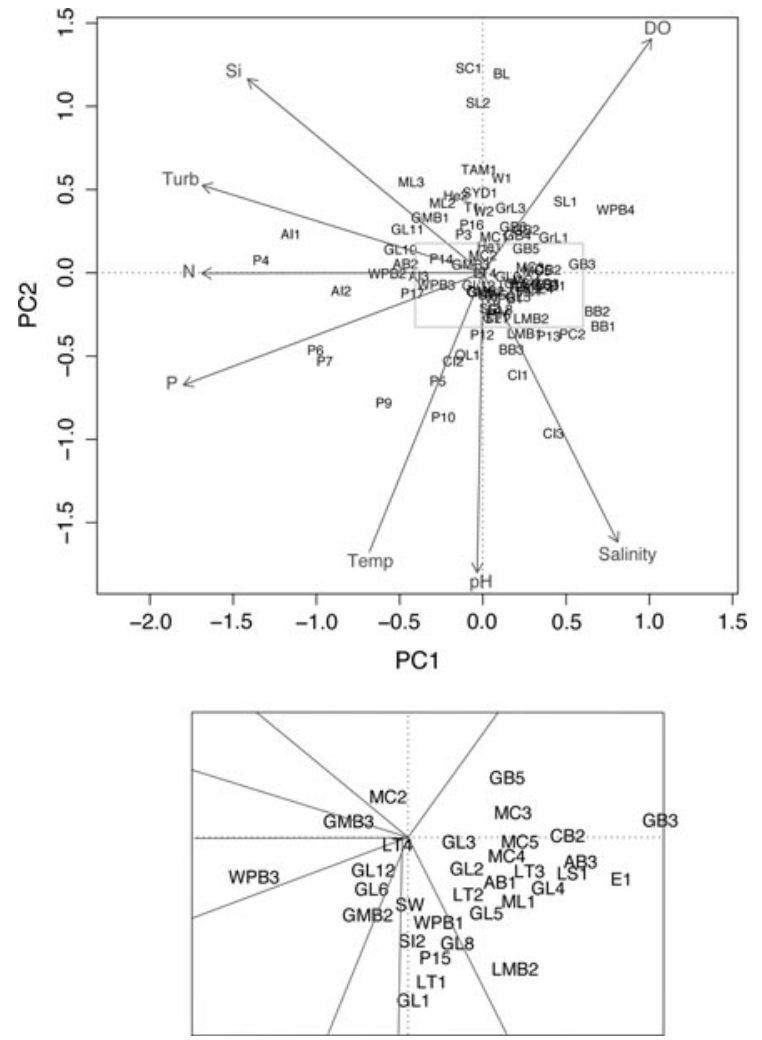

Fig. 2 Principal Components Analysis of the environmental data. Boxed region in the upper panel expanded below. DO dissolved oxygen, $N$ nitrate/nitrite, $P$ phosphate, $S i$ silicate, Temp temperature, Turb turbidity. See Table 3 for site codes and water chemistry information

Table 5 Canonical correspondence analyses of (a) all the environmental variables and (b) forward selected variables only (i.e. nitrate-nitrite, phosphate, salinity and turbidity)

\begin{tabular}{lllll}
\hline Axis & 1 & 2 & 3 & 4 \\
\hline
\end{tabular}

(a) All variables

$\begin{array}{lllll}\text { Eigenvalues } & 0.106 & 0.062 & 0.054 & 0.044 \\ \sum \text { canonical eigenvalues } & 0.390 & & & \\ \sum \text { all eigenvalues } & 2.392 & & & \end{array}$

(b) Forward selected variables

\begin{tabular}{lllll} 
Eigenvalues & 0.103 & 0.054 & 0.043 & 0.038 \\
$\sum$ canonical eigenvalues & 0.238 & & & \\
$\sum$ all eigenvalues & 2.392 & & & \\
\hline
\end{tabular}

$\sum=\operatorname{sum}$

\section{Discussion}

The overall aims of this study were to quantify the relationship between diatom species assemblages and the water chemistry of southeast Australian estuaries and coastal lakes, develop regional inference models, and discuss potential applications of the inference models to ecological and paleoecological studies investigating natural variability and human impacts in coastal ecosystems.

\section{Environmental data and diatom assemblages}

Water chemistry differences between Tasmania and Victoria were primarily related to differences in nutrient and dissolved oxygen concentrations (Table 2). This is likely associated with different land use types, freshwater inputs, influence from the sea and other local and regional factors that influence estuarine nutrient cycling. Mean nitrate + nitrite and phosphate concentrations were nine and six times greater, respectively, in Victorian coastal water bodies compared to Tasmanian sites and no Tasmanian sites had nitrate + nitrite concentrations $\geq 55$ $\mu \mathrm{g} \mathrm{N} \mathrm{L}{ }^{-1}$ or phosphate concentrations $\geq 70 \mu \mathrm{g} \mathrm{P}$ $\mathrm{L}^{-1}$ (Table 2). However, the low median nitrate + nitrite concentration and, to a lesser extent phosphate concentration, in Victorian sites indicated that high nutrient sites were in the minority (Table 2). This was also found by Philibert et al. (2006) in their study of southeast Australian streams, where the majority of sites had low nutrient values. Mean and median dissolved oxygen in Victorian sites was almost half that of Tasmanian sites (Table 2), despite the maximum dissolved oxygen recorded in Victorian sites being greater than in Tasmanian sites. This indicates that, in general, Tasmanian sites have lower nutrient concentrations and higher dissolved oxygen than Victorian sites, which reflects generally better water quality and lower levels of nutrient enrichment in Tasmanian compared to Victorian sites.

The number of taxa identified (399 in total) highlights the wide diversity of diatom taxa in the southeast Australian coastal region. The most common and widespread taxa (Table 4) are considered cosmopolitan species, characteristic of brackish, coastal and marine water bodies (Witkowski et al. 2000) and have been identified in previous Australian coastal diatom studies (e.g. Fluin et al. 2007; Haynes et al. 2007; Saunders et al. 2007; Taffs et al. 2008). 


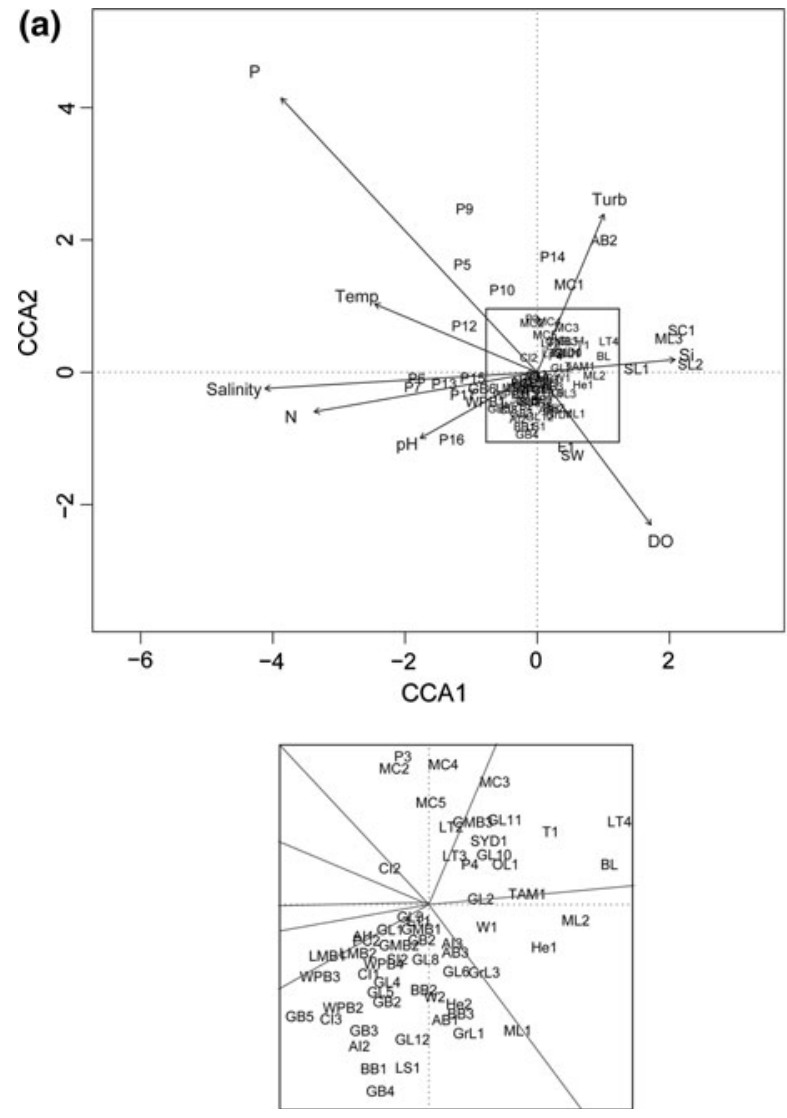

Fig. 3 Canonical Correspondence Analysis of the dataset with all environmental variables. a Sites displayed; b species displayed. Boxed region in the upper panels expanded below.

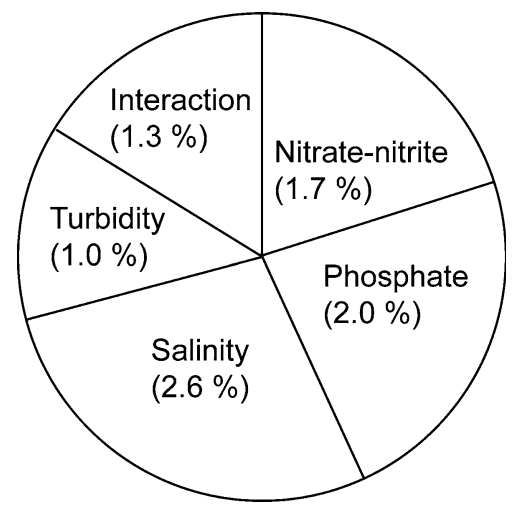

Fig. 4 Summary of Variance Partitioning results

Major environmental gradients and diatom species ecological preferences

This study demonstrated that diatom assemblages in southeast Australian coastal lakes, lagoons and
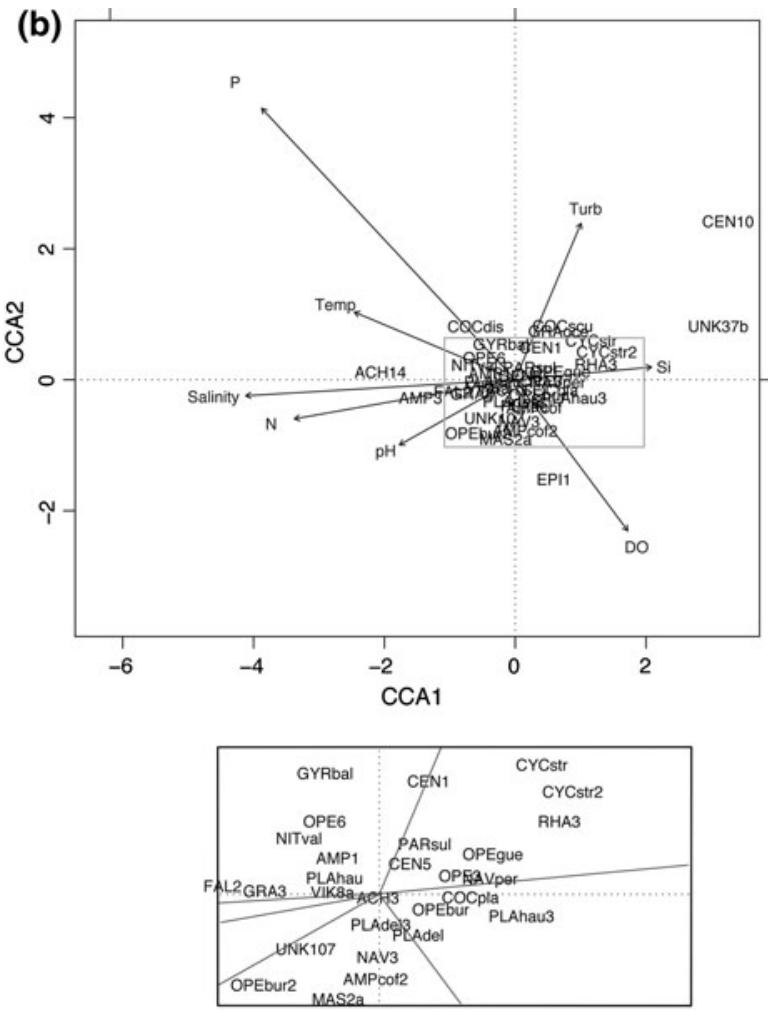

$D O$ dissolved oxygen, $N$ nitrate/nitrite, $P$ phosphate, $S i$ silicate, Temp temperature, Turb turbidity. See Table 4 and Supplementary Material for species names

estuaries are primarily influenced by salinity. Salinity has previously been found to be the overriding environmental variable influencing Australian diatom communities and diatom-salinity (or -conductivity) relationships have been described (e.g. Hodgson et al. 1997; Blinn and Bailey 2001; Gell et al. 2002; Philibert et al. 2006; Saunders et al. 2007; Tibby et al. 2007; Taukulis and John 2009). With the exception of Hodgson et al. (1997) and Saunders et al. (2007), none of these studies were conducted in coastal ecosystems.

Other factors not measured, such as grain size, water depth and light availability, can influence the composition of diatom communities (Stoemer and Smol 1999). For example, water depth has previously been found to have an important influence on benthic diatom communities (e.g. Weckström and Juggins 2005). The influence of depth was minimized in this study as all samples were collected from $1 \mathrm{~m}$ water depth. Similarly, light availability and grain size have 
Fig. 5 Species salinity optima and tolerances, derived by simple weighted averaging

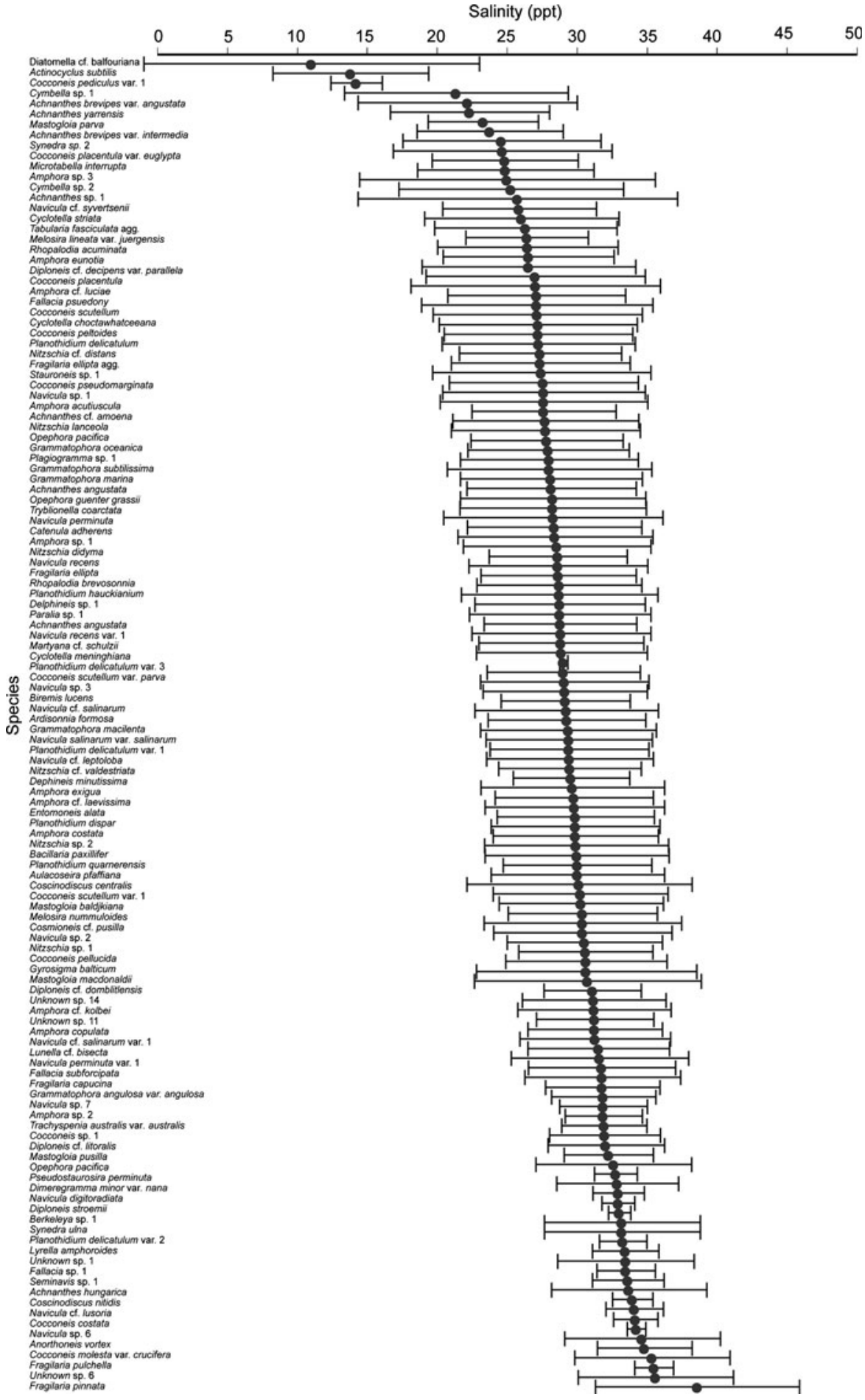

still found salinity is the overriding factor in explaining changes in diatom species assemblages (e.g. Hassan et al. 2009). 


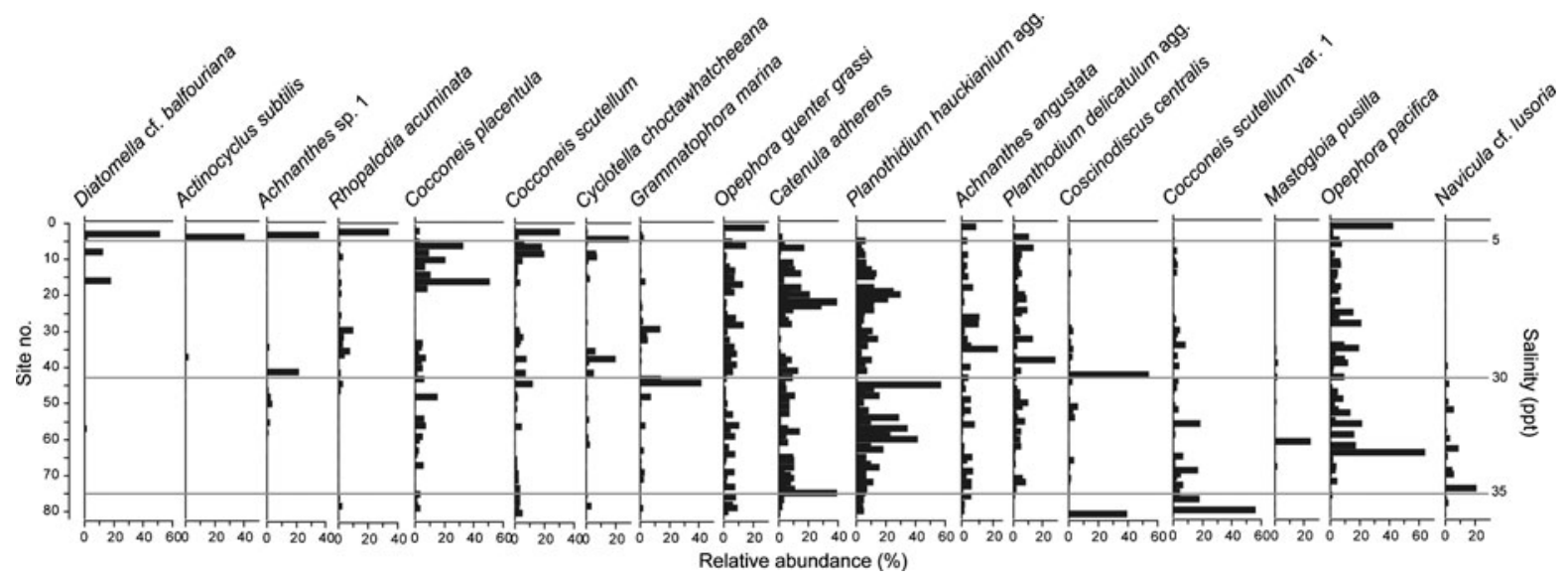

Fig. 6 Occurrence of most dominant ( $\geq 20 \%$ maximum relative abundance) taxa in the dataset. Sites ordered by salinity. Species ordered by their salinity optimum

Table 6 Transfer function results and comparisons with similar studies

\begin{tabular}{llllll}
\hline Model & $r^{2}$ & $r^{2} \mathrm{p}$ & RMSE & RMSEP & References \\
\hline WAinv & 0.73 & 0.54 & $0.07 \log \mathrm{ppt}$ & $0.10 \mathrm{log} \mathrm{ppt}$ & This study \\
WAcla & 0.84 & 0.60 & $0.17 \mathrm{log} \mathrm{ppt}$ & $0.26 \mathrm{log} \mathrm{ppt}$ & Saunders et al. (2007) \\
WAcla & 0.44 & 0.29 & $9.44 \mathrm{ppt}$ & $10.6 \mathrm{ppt}$ & Saunders et al. (2008) \\
WAPLS-2 & 0.87 & 0.75 & $3.12 \mathrm{ppt}$ & $4.42 \mathrm{ppt}$ & Hassan et al. (2009) \\
WAPLS-2 & 0.977 & 0.887 & $0.11 \log \mathrm{g} \mathrm{L}^{-1}$ & $0.246 \log \mathrm{g} \mathrm{L}^{-1}$ salinity & Ryves et al. (2004) \\
WAPLS-2 & 0.84 & - & - & $0.15 \log \mathrm{g} \mathrm{L}^{-1}$ salinity & Clarke et al. (2003) \\
\hline
\end{tabular}

$r^{2} \mathrm{p} r^{2}$ of prediction, $R M S E$ root mean squared error, RMSEP root mean squared error of prediction, ppt parts per thousand

The covariance of environmental gradients in coastal areas and estuaries (particularly with nutrients, e.g. Underwood et al. 1998) may make it difficult to determine the environmental factor to which species are primarily responding to (Ryves et al. 2004). Although it is possible to separate and test the independent effects of different parameters, there is often a large proportion of the variance unexplained (e.g. Ryves et al. 2004; this study). This is likely due to a combination of factors including potential mismatches between time-integrated sedimentary assemblages compared to 'spot' measurements of environmental variables. Additionally, even if spot measurements of environmental variables are repeated they may still fail to adequately capture the variation in physical chemical conditions that affect diatom growth. Therefore, whilst measurements in this study were made both in summer and winter, it is unlikely that the full ranges of the environmental parameters measured was captured at all the sites, which together with unmeasured parameters, contributes to the unexplained portion of diatom species-environment relationships seen in this dataset.

Prior to this study little was known about diatom species ecological preferences in southeast Australian coastal lakes and estuaries. The determination of species optima and tolerances and gradual transitions in species abundances and occurrences along the salinity gradient (Figs. 6,7) allows the identification taxa that are more abundant at particular salinities. Whilst most of these more abundant taxa have been previously described as 'brackish-marine' taxa, this study permits a finer scale discrimination of their salinity preferences. For example, Cocconeis placentula has previously been identified as a brackish, shallow water species (Hodgson et al. 1996; Fluin et al. 2007), and this study is consistent with these observations (i.e. it was most abundant in brackish conditions, 5-30 ppt, Fig. 7). Grammatophora marina (Lyngbye) Kützing, Planothidium hauckianium agg., 

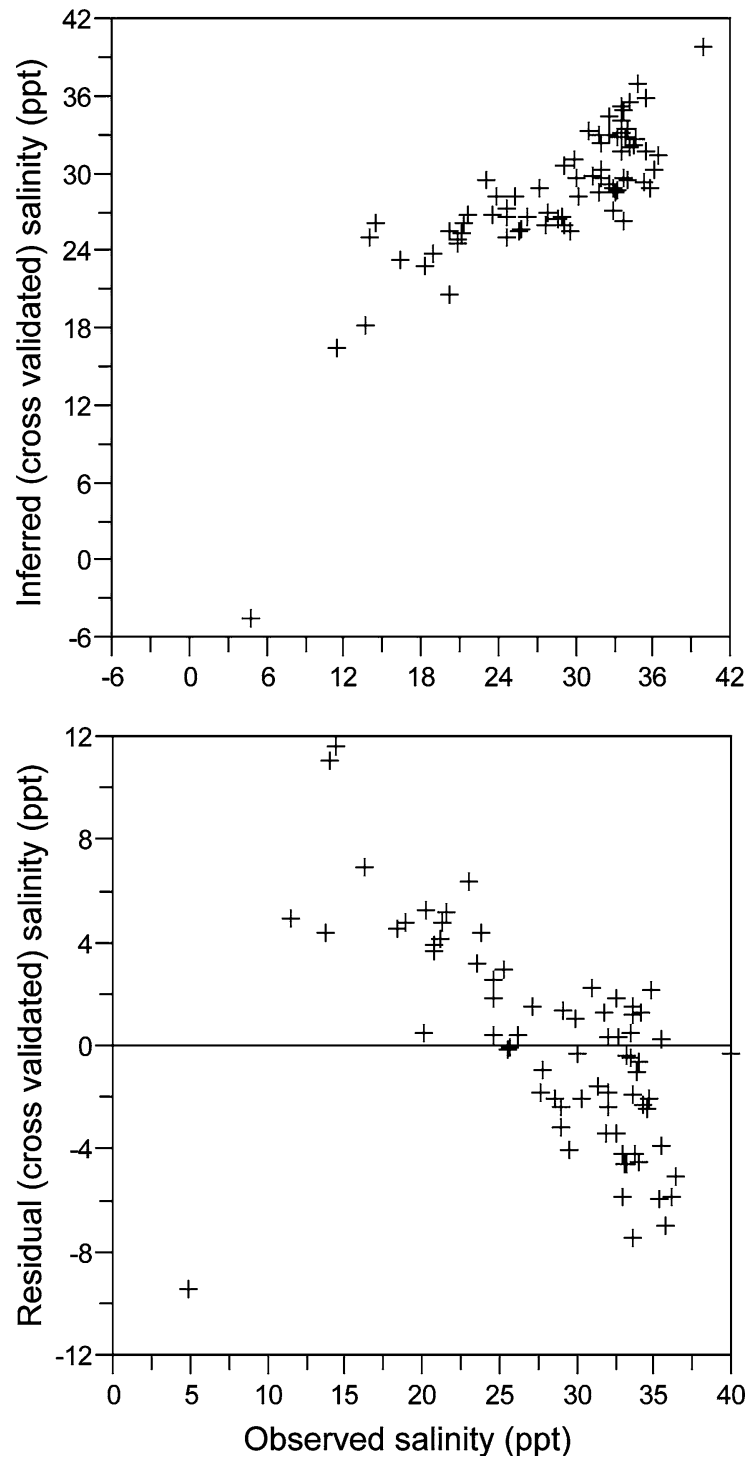

Fig. 7 Plots of observed versus inferred salinity and observed versus residual salinity based on simple WA regression and calibration

Mastogloia pusilla Grunow and Opephora pacifica Petit were most abundant at marine (30-35 ppt) sites (Fig. 7). This agrees with previous descriptions of Grammatophora marina and Opephora pacifica which have been classified as marine taxa (Vos and de Wolf 1993; Fluin et al. 2007). Planothidium hauckianium agg. was very common in the dataset and occurred at most sites. This is a consistent with previous descriptions, which have identified this as a cosmopolitan taxon commonly found in brackish sites, especially on sandy substrates (Witkowski et al. 2000).
Evaluation of the diatom-salinity transfer function

The best performing diatom-salinity transfer function was developed using simple WA with inverse deshrinking. The large number of different waterbody types included in this dataset, ranging from small coastal lakes to large open estuarine systems, provided a wide range of species assemblages distributed along long environmental gradients and a good range of modern analogue assemblages with which to interpret subfossil data. Six sites were identified as outliers and removed (P4, SC1, ML2, ML3, GL10, GL11). All were from low salinity $(\leq 12 \mathrm{ppt})$ sites with very high phosphate concentrations $\left(>450 \mu \mathrm{g} \mathrm{PL}^{-1}\right)$. While the predictive ability of the diatom-salinity transfer function has a $0.09 \log$ ppt (i.e. $4.3 \mathrm{ppt}$ ) error, its performance is better than a previously published transfer function based only on Victorian sites (Saunders et al. 2008). In contrast the Tasmanian transfer function presented by Saunders et al. (2007) has slightly better performance (Table 6) than the current study.

Potential applications of the dataset: ecological and paleoecological investigations of natural variability and human impacts

Environmental impacts on coastal lakes and estuaries in southern Australia are a major management concern. Large-scale environmental problems have arisen due to upstream river regulation, water extraction for irrigation, salinization, enhanced sedimentation rates, modifications of openings to the sea, urbanization of coastal catchments, acidification and eutrophication (SoE 2006; Fluin et al. 2007; Taukulis and John 2009). Management efforts are increasingly focused on alleviating environmental problems ( $\mathrm{SoE}$ 2006). However, historical documentation and water quality monitoring data are usually inadequate for providing the long term perspective needed (SoE 2006). In the absence of long term water quality monitoring, paleoecological methods play a crucial role in establishing 'baseline' conditions and rates of change as they can identify the timing and progressive impact of different human activities and provide a long enough temporal perspective to disentangle natural from anthropogenic-related changes (Willis and Birks 2006). They also provide an opportunity to assess whether ecosystems have crossed critical thresholds (e.g. Smol and Douglas 2007) and take 
into account natural variability, which is essential for being able to evaluate the capacity of coastal ecosystems to adapt and respond to future changes, particularly predicted climate change and further pressure from human activities (Duke et al. 2003; Davis and Koop 2006). The dataset and transfer function developed in this study may be applied to sediment cores collected from coastal lakes and estuaries in southeast Australia to reconstruct past salinity changes and address issues arising from direct (e.g. hydrological modifications to estuary entrances) and indirect (e.g. changes in catchment land use and/or upstream irrigation schemes) human activities, together with the impacts of recent climate change (e.g. reduced rainfall). This provides a tool for understanding whether current conditions are outside the range of natural variability, attribute causes of change (e.g. Saunders et al. 2008) and assist in determining appropriate management strategies.

\section{Conclusion}

This study has presented a new diatom dataset quantifing the relationship between diatom species assemblages and the water chemistry of southeast Australian estuaries and coastal lakes. Salinity explained the most variation in the diatom assemblages and a diatom inference model for reconstructing salinity was developed. This is a potentially valuable tool for monitoring both modern changes in salinity using time-smoothed diatom species assemblages (rather than spot measurements) and reconstructing past salinity changes from sub-fossil species assemblages to identify long term natural variability and human impacts in southeast Australian coastal water bodies.

Acknowledgments This study was funded by an Australian Postgraduate Award, with additional funding from the Australian Institute of Nuclear Science and Engineering, Project Aware and the PADI Foundation. I would particularly like to thank Dr. Dom Hodgson for his advice throughout the project and preparation of this manuscript. I would also like to thank Kathryn Taffs, Kaarina Weckström, John Gibson and Andrew McMinn for project advice and taxonomic assistance; Steve Juggins for statistical advice; and Kate Dziegielewska, Jessie Webb and Sarah Lovibond for field assistance. Special thanks are also due to the two anonymous reviewers of the original version of this manuscript for their very helpful suggestions, which resulted in a much improved presentation of the results.

\section{References}

ANZECC (2000) Australian and New Zealand guidelines for fresh and marine water quality. Australian and New Zealand Environment and Conservation Council, Canberra. Available from http://www.environment.gov.au/ water/quality/nwqms/index.html, Cited Aug 2006

Battarbee RW (1986) Diatom analysis. In: Berglund BE (ed) Handbook of holocene palaeoecology and palaeohydrology. Wiley, New York, pp 527-570

Battarbee RW, Jones VJ, Flower RJ, Cameron NG, Bennion H, Carvalho L, Juggins S (2001) Diatoms. In: Smol JP, Birks HJB, Last WM (eds) Tracking environmental change using lake sediments: terrestrial, algal and siliceous indicators, vol 3. Kluwer, Dordrecht, pp 155-202

Bennion H (2010) European Diatom Database: http://www. ecrc.ucl.ac.uk/index.php/content/view/296/146//(cited 13/ 1/10)

Bennion H, Juggins S, Anderson NJ (1996) Predicting epilimnetic phosphorus concentrations using an improved diatom-based transfer function and its application to lake eutrophication management. Environ Sci Technol 30: 2004-2007

Bigler C, Hall RI (2003) Diatoms as quantitative indicators of July temperature: a validation attempt at century-scale with meteorological data from northern Sweden. Palaeogeogr Palaeoclimatol Palaeoecol 189:147-160

Birks HJB (1998) Numerical tools in paleolimnology-progress, potentialities, and problems. J Paleolimnol 20:307-322

Birks HH, Birks HJB, Flower RJ, Peglar SM, Ramdani M (2001) Recent ecosystem dynamics in nine North African lakes in the CASSARINA Project. Aquat Ecol 35: 461-478

Blinn DW, Bailey PCE (2001) Land-use influence on stream water quality and diatom communities in Victoria, Australia: a response to secondary salinization. Hydrobiologia 466:231-244

BOM (2009) http://www.bom.gov.au. Cited Nov 2008

Clarke A, Juggins S, Conley DJ (2003) A 150-year reconstruction of the history of coastal eutrophication in Roskilde Fjord, Denmark. Mar Pol Bul 46:1615-1629

Davis JR, Koop K (2006) Eutrophication in Australian rivers, reservoirs and estuaries - a southern hemisphere perspective on the science and its implications. Hydrobiologia 559:23-76

Duke N, Lawn PT, Roelfsema CM, Zahmel KN, Pedersen DK, Harris C, Steggles N, Tack C (2003) Assessing historical change in coastal environments: Port Curtis, Fitzroy River Estuary and Moreton Bay Regions. Report to the Cooperative Research Centre for Coastal Zone Estuary and Water Management. Brisbane: Marine Botany Group, Centre for Marine Studies, University of Queensland

Ellegaard M, Clarke A, Reuss N, Drew S, Weckström K, Juggins S, Anderson NJ, Conley DJ (2006) Multi-proxy evidence of long-term changes in ecosystem structure in a Danish marine estuary, linked to increased nutrient loading. Estuar Coast Shelf Sci 68:567-578

Eriksen R (1997) A practical manual for the determination of salinity, dissolved oxygen, and nutrients in seawater. Antarctic Co-operative Research Centre Report No. 11, Hobart, Australia 
Fluin J, Gell PA, Haynes D, Tibby J, Hancock G (2007) Palaeolimnological evidence for the independent evolution of neighbouring terminal lakes, the Murray Darling Basin, Australia. Hydrobiologia 591:117-134

Fritz SC, Juggins S, Battarbee RW, Engstrom DR (1991) Reconstruction of past changes in salinity and climate using a diatom-based transfer function. Nature 352: 706-708

Gasse F, Juggins S, Khelifa LB (1995) Diatom-based transfer functions for inferring past hydrochemical characteristics of African lakes. Palaeogeogr Palaeoclimatol Palaeoecol 117:31-54

Gehrels WR, Szkornik K, Bartholdy J, Kirby JR, Bradley SL, Marshall WA, Heinemeier J, Pedersen JBT (2006) Late Holocene sea-level changes and isostasy in western Denmark. Quat Int 66:208-302

Gell PA, Sluiter IR, Fluin J (2002) Seasonal and interannual variations in diatom assemblages in Murray River connected wetlands in north-west Victoria, Australia. Mar Freshw Res 53:981-992

Glew JR (1991) Miniature gravity corer for recovering short gravity cores. J Paleolimnol 5:285-287

Hassan GS, Espinosa MA, Isla FI (2007) Dead diatom assemblages in surface sediments from a low impacted estuary: the Queque'n Salado river, Argentina. Hydrobiologia 579:257-270

Hassan GS, Espinosa MA, Isla FI (2009) Diatom-based inference model for paleosalinity reconstructions in estuaries along the northeastern coast of Argentina. Palaeogeogr Palaeoclimatol Palaeoecol 275:77-91

Haynes D, Gell PA, Tibby J, Hancock G, Goonan P (2007) Against the tide: the freshening of naturally saline coastal lakes, southeastern South Australia. Hydrobiologia 591:165-183

Hill MO (1973) Diversity and evenness: a unifying notation and its consequences. Ecology 54:427-432

Hodgson DA, Tyler PA, Vyverman W (1996) The palaeolimnology of Lake Fidler, a meromictic lake in south west Tasmania and the significance of recent human impact. J Paleolimnol 18:313-333

Hodgson DA, Vyverman WG, Tyler PA (1997) Diatoms of meromictic lakes adjacent to the Gordon River, and of the Gordon River estuary in south-west Tasmania. In: LangeBerlot H, Kociolek P (eds) Bioliotheca diatomologica band 35. Gebruder Borntraeger, Berlin

Horton BP, Corbett R, Culver SJ, Edwards RJ, Hillier C (2006) Modern saltmarsh diatom distributions of the Outer Banks, North Carolina, and the development of a transfer function for high resolution reconstructions of sea level. Estuar Coast Shelf Sci 69:381-394

John J (1983) The diatom flora of the Swan River Estuary Western Australia, vol 64. Bibliotheca Phycologica, Vaduz

Juggins S (1992) Diatoms in the Thames Estuary, England: ecology, paleoecology, and salinity transfer function. In: Lange-Bertalot H (ed) Bibliotheca Diatomologica, vol 25. J. Cramer, Berlin, pp 1-216

Juggins S (2003) C2 user guide. Software for ecological and palaeoecological data analysis and visualisation. University of Newcastle, Newcastle Upon Tyne
Juggins S (2004) Monitoring long-term trends in eutrophication and nutrients in the coastal zone. Available from http://Craticula.ncl.ac.uk/Molten/jsp/

Kingston JC, Birks HJB, Uutala AJ, Cumming BF, Smol JP (1992) Assessing trends in fishery resources and lake water aluminum from paleolimnological analyses of siliceous algae. Can J Fish Aquat Sci 49:116-127

Lim DSS, Smol JP, Douglas MSV (2007) Diatom assemblages and their relationship to lakewater nitrogen levels and other limnological variables from 36 lakes and ponds on Banks Island, N.W.T., Canadian Arctic. Hydrobiologia 586:191-211

Philibert A, Gell PA, Newall P, Chessman B, Bate N (2006) Development of diatom-based tools for assessing stream water quality in south-eastern Australia: assessment of environmental transfer functions. Hydrobiologia 572: 103-114

Reid MA, Tibby JC, Penny D, Gell PA (1995) The use of diatoms to assess past and present water quality. Aust $\mathrm{J}$ Ecol 20:57-64

Ryves DB, Clarke AL, Appleby PG, Amsinck SL, Jeppesen E, Landkildehus F, Anderson NJ (2004) Reconstructing the salinity and environment of the Limfjord and Vejlerne Nature Reserve, Denmark, using a diatom model for brackish lakes and fjords. Can J Fish Aquat Sci 61: 1988-2006

Saunders KM, McMinn A, Roberts D, Hodgson DA, Heijnis H (2007) Recent human-induced salinity changes in Ramsar-listed Orielton lagoon, southeast Australia. Aquat Conserv Mar Freshw Ecosyst 17:51-70

Saunders KM, Hodgson DA, Harrison J, McMinn A (2008) Palaeoecological tools for improving the management of coastal ecosystems: a case study from Lake King (Gippsland Lakes) Australia. J Paleolimnol 40:33-47

Smol JP, Douglas MSV (2007) Crossing the final ecological threshold in high Arctic ponds. Proc Nat Acad Sci USA 104:12395-12397

SoE (2006) State of the environment report. Australian Commonwealth Government Department of Environment and Heritage, available at: http://www.deh.gov.au/soe/2006/

Sonneman JA, Sincock A, Fluin J, Reid MA, Newall P, Tibby JC, Gell P (2000) An illustrated guide to common stream diatom species from temperate Australia. Cooperative Research Centre for Freshwater Ecology, Albury

Stoemer EF, Smol JP (1999) The diatoms: applications for the environmental and earth sciences. Cambridge University Press, Cambridge, pp 352-373

Taffs KH (2005) Diatoms of Northern New South Wales, Australia. Unpublished dataset. Southern Cross University, Australia

Taffs KH, Farago LJ, Heijnis H, Jacobsen G (2008) A diatombased Holocene record of human impact from a coastal environment: Tuckean Swamp, eastern Australia. J Paleolimnol 39:71-82

Taukulis FE, John J (2009) Development of a diatom-based transfer function for lakes and streams severely impacted by secondary salinity in the south-west region of Western Australia. Hydrobiologia 626:129-143

R Development Core Team (2006) R: a language and environment for statistical computing. $\mathrm{R}$ Foundation for 
Statistical Computing, Vienna, Austria. ISBN 3-900051-

07-0. Available from http://www.R-project.org

ter Braak CJF (1995) Ordination. In: Jongman RHG, ter Braak CJF, van Tongeren OFR (eds) Data analysis in community and landscape ecology. Cambridge University Press, Cambridge, pp 91-173

ter Braak CJF, Smilauer P (2002) CANOCO Reference manual and CanoDraw for Windows user's guide: software for canonical community ordination (version 4.5). Microcomputer Power, Ithaca

Tibby J, Gell PA, Fluin J, Sluiter IR (2007) Diatom-salinity relationships in wetlands: assessing the influence of salinity variability on the development of inference models. Hydrobiologia 591:207-218

Underwood GJC, Phillips J, Saunders K (1998) Distribution of estuarine benthic diatom species along salinity and nutrient gradients. Eur J Phycol 33:173-183

Vos PC, de Wolf H (1993) Reconstruction of sedimentary environments in Holocene coastal deposits of the southwest Netherlands; the Poortvliet boring, a case study of paleoenvironmental diatom research. Hydrobiologia 269/ 270:297-306

Vyverman W, Vyverman R, Hodgson D, Tyler P (1995) Diatoms from Tasmanian mountain lakes: a reference data-set
(TASDIAT) Bibliotheca Diatomologica, Band 33. J. Cramer in der Gebrüder Borntraeger Verlagsbuchhandlung, Berlin, pp 1-193

Weckström K, Juggins S (2005) Coastal diatom-environment relationships from the Gulf of Finland, Baltic Sea. J Phycol 42:21-35

Weckström K, Juggins S, Korhola A (2004) Quantifying background nutrient concentrations in coastal waters: a case study from an urban embayment of the Baltic Sea. Ambio 33:324-327

Willis KJ, Birks JB (2006) What is natural? The need for a long-term perspective in biodiversity conservation. Science 314:1261-1264

Wilson SE, Cumming BF, Smol JP (1996) Assessing the reliability of salinity inference models from diatom assemblages: an examination of a 219-lake data set from western North America. Can J Fish Aquat Sci 53:1580-1594

Witkowski A, Lange-Berlot H, Metzeltin D (2000) Diatom flora of marine coasts. In: Lange-Berlot $\mathrm{H}$ (ed) Iconographica diatomologica: annotated diatom micrographs, vol 7. A. R. G. Ganter Verlag K. G, Berlin 